\title{
Effect of the measurement on the decay rate of a quantum system
}

\author{
Brahim Elattari ${ }^{1,2}$ and S.A. Gurvitz ${ }^{3}$ \\ ${ }^{1}$ Weizmann Institute of Science, Department of Condensed Matter Physics \\ 76100 Rehovot, Israel \\ ${ }^{2}$ Université Chouaïb Doukkali, Faculté des Sciences, El Jadida, Morocco \\ ${ }^{3}$ Weizmann Institute of Science, Department of Particle Physics \\ 76100 Rehovot, Israel
}

\begin{abstract}
We investigated the electron tunneling out of a quantum dot in the presence of a continuous monitoring by a detector. It is shown that the Schrödinger equation for the whole system can be reduced to new Bloch-type rate equations describing the time-development of the detector and the measured system at once. Using these equations we find that the continuous measurement of the unstable system does not affect its exponential decay, $\exp (-\Gamma t)$, contrary to expectations based on the Quantum Zeno effect. However, the width of the energy distribution of the tunneling electron is no more $\Gamma$, but increases due to the decoherence, generated by the detector.
\end{abstract}

PACS: 73.23.Hk.03.65.Bz.73.23.-b

It was suggested that an unstable quantum system slows down its decay rate under frequent or continuous observations [1]. This phenomenon, known as the quantum Zeno effect, is believed to be related to the projection postulate in the theory of quantum measurements [2]. Indeed, in the standard example of two-level systems, the probability of a quantum transition from an initially occupied unstable state is $Q(\Delta t)=a(\Delta t)^{2}$. If we assume that $\Delta t$ is the measurement time, which consists in projecting the system onto the initial state, then after $N$ successive measurements the probability of finding the unstable system in its initial state, at time $t=N \Delta t$, is $P(t)=\left[1-a(\Delta t)^{2}\right]^{(t / \Delta t)}$. It follows from this result that $P(t) \rightarrow 1$ for $\Delta t \rightarrow 0$, i.e. suppression of quantum transition.

Originally quantum Zeno effect has been considered as a slowing down of the decay rate [i] of quantum systems in which a discrete initial state is coupled to a continuum of final states. This coupling leads to an irreversible exponential decay from the discrete state to the continuum of states. This situation is very often encountered in physics, as for instance, the $\alpha$-decay of a nucleus, the spontaneous emission of a photon by an excited atom, the photoelectric effect, and so on. But from the theoretical and experimental point of view, the effort has been mainly concentrated on quantum transitions between isolated levels [3] characterized by an oscillatory behavior between the different states. In this latter case the slowing down of the transition rate has, indeed, been found. However, this was attributed to the decoherence generated by the detector without an explicit involvement of the projection postulate [4,5]. On the other hand, the slowing down of the exponential decay rate still remains a controversial issue, despite the fact that it is extensively studied [6 9] and further investigations are clearly desirable.

In this letter we focus our attention on the quantum
Zeno effect in exponentially decaying systems, using a microscopic description which includes the measurement devices. The latter is an essential point missed in many studies. This work is motivated by the distinct difference between continuum energy levels and discrete levels, stated above, as well as by the theoretical and experimental importance of the subject. We also propose an experimental set up which is within reach of nowadays experimental techniques and within which the quantum Zeno effect for exponentially decaying systems can be investigated. Our results showed that while the decay rate of the quantum unstable system is unaffected by the measurement the energy distribution of the emitted particles can be strongly affected.
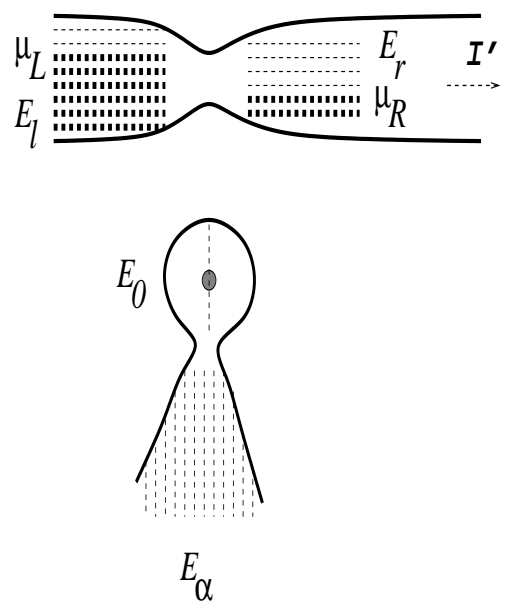

Fig. 1: The point-contact detector near the quantum dot. The energy level $E_{0}$ of the dot is occupied by an electron, which tunnels to continuous states $E_{\alpha}$ of the reservoir. $\mu_{L}$ and $\mu_{R}$ are the Fermi levels in the emitter and the collector, respectively.

Let us consider an electron tunneling out of a quantum dot to a reservoir of very dense (continuum) states, 
$E_{\alpha}$. The dot is placed near a quantum point-contact connected with two separate reservoirs (Fig. 1). The reservoirs are filled up to the Fermi levels $\mu_{L}$ and $\mu_{R}$, respectively. Therefore the current $I=e^{2} T V / 2 \pi$ flows from the left (emitter) to the right reservoir (collector), where $T$ is the transmission coefficient of the point-contact and $\mathrm{eV}=\left(\mu_{L}-\mu_{R}\right)$ is the bias voltage [10]. (We consider the case of zero temperature). However, when the dot is occupied, Fig. 1, the transmission coefficient of the point-contact decreases $\left(T^{\prime}<T\right)$ due to Coulomb repulsion generated by the electron inside the dot. Respectively, the current through the quantum dot diminishes, $I^{\prime}<I$. Thus, the point-contact does represent a detector, which monitors the occupation of the quantum dot. Actually, such a point-contact detector has been successfully used in different experiments [11]. Notice that the current variation $\left(I-I^{\prime}\right)$ can be a macroscopic quantity if the applied voltage $V$ is large enough. The dynamics of the entire system is determined by the many-body time-dependent Schrödinger equation $i|\dot{\Psi}(t)\rangle=H|\Psi(t)\rangle$, where the total Hamiltonian consists of three components $H=H_{Q D}+H_{P C}+H_{\text {int }}$, describing the quantum dot, the point-contact detector, and their mutual interaction, respectively. These three parts can be written in the form of tunneling Hamiltonians, as

$$
\begin{aligned}
H_{Q D} & =E_{0} c_{0}^{\dagger} c_{0}+\sum_{\alpha} E_{\alpha} c_{\alpha}^{\dagger} c_{\alpha}+\sum_{\alpha}\left[\Omega_{\alpha} c_{\alpha}^{\dagger} c_{0}+H . c .\right] \\
H_{P C} & =\sum_{l} E_{l} c_{l}^{\dagger} c_{l}+\sum_{r} E_{r} c_{r}^{\dagger} c_{r}+\sum_{l, r}\left[\Omega_{l r} c_{l}^{\dagger} c_{r}+H . c .\right] \\
H_{\text {int }} & =\sum_{l, r}\left[\delta \Omega_{l r} c_{l}^{\dagger} c_{r}+H . c .\right] c_{0}^{\dagger} c_{0}
\end{aligned}
$$

Where the operators $c_{i}^{+}\left(c_{i}\right)$ correspond to the creation (annihilation) of an electron in state $i$. The $\Omega_{\alpha}$ and $\Omega_{l r}$ are the hopping amplitudes between the states $E_{0}, E_{\alpha}$ and $E_{l}, E_{r}$ respectively. These amplitudes are found to be directly related to the tunneling rate of the electron out of the quantum dot $(\Gamma)$ and to the penetration coefficient of the point-contact $(T)$ as $\Gamma=2 \pi\left|\Omega_{\alpha}\right|^{2} \rho$ and $T=(2 \pi)^{2}\left|\Omega_{l r}\right|^{2} \rho_{L} \rho_{R}$, respectively. Here $\rho$ are the density of states in the corresponding reservoirs. The quantity $\delta \Omega_{l r}=\Omega_{l r}^{\prime}-\Omega_{l r}$ represents the variation of the pointcontact hopping amplitude, when the dot is occupied. In our derivations we assume that $\Omega$ and $\rho$ are weakly energy dependent and $\left(\mu_{L}-\mu_{R}\right) \gg \Omega^{2} \rho$. The latter condition is necessary for the exact solubility of the model.

Consider the entire system in the initial condition, corresponding to occupied quantum dot and filled reservoirs up to Fermi levels $\mu_{L}$ and $\mu_{R}$, Fig. 1, denoted by $|\Psi(0)\rangle=|0\rangle$. This state is not stable: the Hamiltonian (11) requires it to decay to continuum states having the form $c_{i}^{\dagger} c_{i_{1}}^{\dagger} \cdots c_{j} c_{j_{1}} \cdots|0\rangle$. In general, the total wave-function at time $t$ can be written as

$$
\begin{array}{r}
|\Psi(t)\rangle=\left[b_{0}(t)+\sum_{l, r} b_{l r}(t) c_{r}^{\dagger} c_{l}+\sum_{\alpha} b_{\alpha}(t) c_{\alpha}^{\dagger} c_{0}\right. \\
\left.+\sum_{\alpha, l, r} b_{\alpha l r}(t) c_{r}^{\dagger} c_{\alpha}^{\dagger} c_{l} c_{0}+\cdots\right]|0\rangle .
\end{array}
$$

Where $b(t)$ are the probability amplitudes of finding the system in the state defined by the corresponding creation and annihilation operators. Using these amplitudes one finds the reduced density-matrix by tracing out the irrelevant degrees of freedom. This density matrix will give us all probability distributions describing the behavior of the entire system. For instance, $\sigma_{00}^{(0)}(t)=\left|b_{0}(t)\right|^{2}$, is the probability of finding the system in the initial state at time $t, \sigma_{00}^{(1)}(t)=\sum_{l, r}\left|b_{l r}(t)\right|^{2}$ is the probability of finding one electron in the collector and the quantum dot is occupied, $\sigma_{\alpha \alpha}^{(0)}(t)=\sum_{\alpha}\left|b_{\alpha}(t)\right|^{2}$ is the probability for the electron to tunnel out of the dot into level $E_{\alpha}$ and no electron arriving the collector, and so on. In general, the total probability for the electron to occupy the dot is $\sigma_{00}(t)=\sum_{n} \sigma_{00}^{(n)}(t)$, and the probability of tunneling into level $E_{\alpha}$ is $\sigma_{\alpha \alpha}(t)=\sum_{n} \sigma_{\alpha \alpha}^{(n)}(t)$. Here the subscript $n$ denotes the number of electrons reaching the collector at time $t$. The corresponding off-diagonal density-matrix elements $\sigma_{0 \alpha}=\sigma_{\alpha 0}^{*}$ describe the electron in the linear superposition of the states $E_{0}$ and $E_{\alpha}$.

In order to find the amplitudes $b(t)$, we substitute Eq. (2) into the time dependent Scrödinger equation and use the Laplace transform $\tilde{b}(E)=\int_{0}^{\infty} b(t) \exp (i E t) d t$. Then we find an infinite set of algebraic equations for the amplitudes $\tilde{b}(E)$, given by

$$
\begin{aligned}
& E \tilde{b}_{0}-\sum_{\alpha} \Omega_{\alpha} \tilde{b}_{\alpha}-\sum_{l, r} \Omega_{l r}^{\prime} \tilde{b}_{l r}=i \\
& \left(E+E_{0}-E_{\alpha}\right) \tilde{b}_{\alpha}-\Omega_{\alpha} \tilde{b}_{0}-\sum_{l, r} \Omega_{l r} \tilde{b}_{\alpha l r}=0, \\
& \left(E+E_{l}-E_{r}\right) \tilde{b}_{l r}-\sum_{\alpha} \Omega_{\alpha} \tilde{b}_{\alpha l r} \\
& -\Omega_{l r}^{\prime} \tilde{b}_{0}-\sum_{l^{\prime}, r^{\prime}} \Omega_{l^{\prime} r^{\prime}}^{\prime} \tilde{b}_{l l^{\prime} r r^{\prime}}=0
\end{aligned}
$$

It is very important that the tracing of the reservoir variables can be carried out directly in Eqs. (3) without their explicit solutions. As a result, Eqs. (3) are converted to the Bloch-type equations for the reduced density-matrix $\sigma_{i j}^{(n)}(t)$. Such a technique has been derived in [5. 12]. In this paper we generalize it by converting Eqs. (3) into rate equations without tracing over all the continuum states. We, thus, obtain generalized Bloch-type equations which determine the energy distribution of the tunneling particles. In the following we outline this derivation, relegating the technical details to a more extended publication. 
First, we replace each of the sums in Eqs. (3) by an integral, $\sum_{k} \rightarrow \int \rho\left(E_{k}\right) d E_{k}$ which can be treated analytically. The Eq. (3a), for instance, after replacing in it the amplitudes $\tilde{b}_{\alpha}$ and $\tilde{b}_{l r}$ by the corresponding expressions obtained from Eqs. (3b), (3c), becomes

$$
\begin{aligned}
& \left(E-\int \frac{\Omega_{\alpha}^{2} \rho\left(E_{\alpha}\right) d E_{\alpha}}{E+E_{0}-E_{\alpha}}\right. \\
& \left.-\int \frac{\Omega^{\prime 2}{ }_{l r} \rho_{L}\left(E_{l}\right) \rho_{R}\left(E_{r}\right) d E_{l} d E_{r}}{E+E_{l}-E_{1}-E_{r}}\right) \tilde{b}_{0}(E)+\mathcal{F}=i,
\end{aligned}
$$

where $\mathcal{F}$ denotes the terms in which the amplitudes $\tilde{b}$ cannot be factorized out the integrals. These terms vanish when the integration limits are extended to infinity (the large bias limit, $\left.\left(\mu_{L}-\mu_{R}\right) \gg \Omega_{L, R}^{2} \rho_{L, R}\right)$. This is due to the fact that the singularities of the amplitudes $\tilde{b}$, as functions of the variables $E_{l, r, \alpha}$, lie on the same side of the integration contour. The remaining integrals in Eq. (4) can be splited into a sum of singular and principal parts. The singular parts yield $i \Gamma / 2+i D^{\prime} / 2$, where $D^{\prime}=(2 \pi)\left|\Omega_{l r}^{\prime}\right|^{2} \rho_{L} \rho_{R}\left(\mu_{l}-\mu_{R}\right)$. While the principal parts induce a shift of energy which is merely absorbed by a redefinition of the energy levels. Performing the same procedure with all other equations (3), we reduce them to the following system of equations for the amplitudes $\tilde{b}(E)$ [. 12 .

$$
\begin{aligned}
& \left(E+i \frac{D^{\prime}}{2}+i \frac{\Gamma}{2}\right) \tilde{b}_{0}(E)=i \\
& \left(E+E_{0}-E_{\alpha}+i \frac{D}{2}\right) \tilde{b}_{\alpha}(E)-\Omega_{\alpha} \tilde{b}_{0}(E)=0 \\
& \left(E+E_{l}-E_{r}+i \frac{D^{\prime}}{2}+i \frac{\Gamma}{2}\right) \tilde{b}_{l r}(E)-\Omega_{l r}^{\prime} \tilde{b}_{l r}(E)=0 \\
& \ldots \ldots \ldots
\end{aligned}
$$

where $D=(2 \pi)\left|\Omega_{l r}\right|^{2} \rho_{L} \rho_{R}\left(\mu_{l}-\mu_{R}\right)$. In order to transform Eq. (5) to equations for the density-matrix we multiply each of them by the corresponding complex conjugate amplitude $b^{*}\left(E^{\prime}\right)$. For instance by multiplying Eq. (5b by $b_{\alpha}^{*}\left(E^{\prime}\right)$ and subtracting its complex conjugated equation multiplied by $b_{\alpha}(E)$ we obtain

$$
\begin{aligned}
& \left(E-E^{\prime}+i D\right) b_{\alpha}(E) b_{\alpha}^{*}\left(E^{\prime}\right) \\
& \quad=\Omega_{\alpha}\left[b_{0}(E) b_{\alpha}^{*}\left(E^{\prime}\right)-b_{0}^{*}\left(E^{\prime}\right) b_{\alpha}\left(E^{\prime}\right)\right]
\end{aligned}
$$

It is quite easy to see that the inverse Laplace transform turns this equation to the following one for the densitymatrix

$$
\dot{\sigma}_{\alpha \alpha}^{(0)}=-D \sigma_{\alpha \alpha}^{(0)}+i \Omega_{\alpha}\left(\sigma_{0 \alpha}^{(0)}-\sigma_{\alpha 0}^{(0)}\right)
$$

Proceeding in the same way with all other equations (5) and integrating over the continuum states of the collector and the emitter, we obtain the following infinite set of equations for the density matrix $\sigma(t)$

$$
\begin{aligned}
& \dot{\sigma}_{00}^{(n)}=-\left(\Gamma+D^{\prime}\right) \sigma_{00}^{(n)}+D^{\prime} \sigma_{00}^{(n-1)} \\
& \dot{\sigma}_{\alpha \alpha}^{(n)}=-D \sigma_{\alpha \alpha}^{(n)}+D \sigma_{\alpha \alpha}^{(n-1)}+i \Omega_{\alpha}\left(\sigma_{0 \alpha}^{(n)}-\sigma_{\alpha 0}^{(n)}\right) \\
& \dot{\sigma}_{\alpha 0}^{(n)}=i\left(E_{0}-E_{\alpha}\right) \sigma_{\alpha 0}^{(n)}-i \Omega_{\alpha} \sigma_{00}^{(n)}-\frac{\Gamma+D+D^{\prime}}{2} \sigma_{\alpha 0}^{(n)} \\
& \quad+\sqrt{D D^{\prime}} \sigma_{\alpha 0}^{(n-1)} .
\end{aligned}
$$

Eqs. (8) are a generalization of the previously derived Bloch-type rate-equations for quantum transport in mesoscopic systems [5 12. They have a clear physical interpretation. Consider for example Eq. (8a) for the probability of finding the electron inside the dot and $n$ electrons in the collector. It decreases due to oneelectron hopping to the collector (with rate $D^{\prime}$ ), or due to the electron tunneling out of the dot (with rate $\Gamma$ ). These processes are described by the first ("loss") term in Eq. (8a). On the other hand, there exists the opposite ("gain") process when the state with $(n-1)$ electrons in the collector converts into the state with $n$ electrons in the collector. It also takes place due to penetration of one electron through the point-contact with the same rate $D^{\prime}$ (the second term in Eq. (8a)).

The evolution of the off-diagonal density-matrix elements is given by Eq. (8d). It can be interpreted in the same way as the rate equation for the diagonal terms. Notice, however, the difference between the "loss" and the "gain" terms. The latter can appear only due to coherent transition of the whole linear superposition [5, 12.

Since our rate equations distinguish between different continuum states $\left(E_{\alpha}\right)$, we can find the energy distribution of the tunneling electron by tracing out the detector states $n$ in Eqs. (8). As a result we obtain the following final equations for the electron density-matrix $\sigma(t)$

$$
\begin{aligned}
\dot{\sigma}_{00} & =-\Gamma \sigma_{00} \\
\dot{\sigma}_{\alpha \alpha} & =i \Omega_{\alpha}\left(\sigma_{0 \alpha}-\sigma_{\alpha 0}\right) \\
\dot{\sigma}_{\alpha 0} & =i\left(E_{0}-E_{\alpha}\right) \sigma_{\alpha 0}-i \Omega_{\alpha} \sigma_{00}-\frac{\Gamma+\Gamma_{d}}{2} \sigma_{\alpha 0} .
\end{aligned}
$$

Here $\Gamma_{d}=\left(\sqrt{D}-\sqrt{D^{\prime}}\right)^{2}$ is the decoherence rate [5].

It is instructive to compare Eqs. (9) with the similar Bloch-type equations describing quantum transitions between two isolated levels [5, 13]. In the case of isolated levels $\left(E_{1}\right.$ and $\left.E_{2}\right)$, the equations for the density-matrix $\sigma$ are symmetric with respect to $E_{1}$ and $E_{2}$. Whereas in the case of transition between the isolated $\left(E_{0}\right)$ and the continuum states $\left(E_{\alpha}\right)$ the corresponding symmetry, between $E_{0}$ and $E_{\alpha}$, is broken as can be seen, for example, in the equation for the off-diagonal term $\sigma_{0 \alpha}$ where the coupling with $\sigma_{\alpha \alpha}$ is missing. Eq. (9d).

The probability of finding the electron inside the dot is obtained directly from Eq. (9a) given by

$$
\sigma_{00}(t)=\exp (-\Gamma t) .
$$

It means that the continuous monitoring of the unstable system does not slow down its exponential decay. Nevertheless, it can be shown that the energy distribution of 
the tunneling electron, $P\left(E_{\alpha}\right) \equiv \sigma_{\alpha \alpha}(t \rightarrow \infty)$, is affected. Indeed, by solving Eqs. (9) in the limit of $t \rightarrow \infty$ we find a Lorentzian distribution centered about $E_{\alpha}=E_{0}$ :

$$
P\left(E_{\alpha}\right)=\frac{\left|\Omega_{\alpha}\right|^{2}}{\Gamma} \frac{\Gamma+\Gamma_{d}}{\left(E_{0}-E_{\alpha}\right)^{2}+\left(\Gamma+\Gamma_{d}\right)^{2} / 4} .
$$

If there is no coupling with the detector, $\Gamma_{d}=0$, the Lorentzian width (the line-width) $\Gamma$ is exactly the inverse life-time of the quasi-stationary state, Eq. (10). However, it follows from Eq. (11) that the measurement results in a broadening of the line-width, which becomes $\Gamma+\Gamma_{d}$ due to the decoherence generated by the detector. At first sight this result might look very surprising. Indeed, it is commonly accepted that the line-width does correspond to the life-time. Yet, we demonstrated here that it might not be the case when the system interacts with an environment (the detector). To understand this result, one might think of the following argument. Due to the measurement, the energy level $E_{0}$ suffers an additional broadening of the order of $\Gamma_{d}$. However, this broadening does not affect the decay rate of the electron $\Gamma$, since the exact value of $E_{0}$ relative to $E_{\alpha}$ is irrelevant to the decay process. In contrast, the probability distribution $P\left(E_{\alpha}\right)$ is affected because it does depend on the position of $E_{0}$ relative to $E_{\alpha}$ as it can be seen in Eq. (11).

Although our result has been proved for a specific detector, we expect it to be valid for the general case, provided that the density of states $\rho$ and the transition amplitude $\Omega$ vary slowly with energy. This condition is sufficient to obtain a pure exponential decay of the state $E_{0}$. On the contrary, if $\rho_{\alpha}\left(E_{\alpha}\right)$ or $\Omega_{\alpha}$ depend sharply on energy, then the integrals in Eq. (四) yield additional $E$-dependent terms that modify both the exponential dependence of the decay probability, Eq. (10) and the energy distribution, Eq. (11). As a result, the measurement process could hinder the decay rate 14 .

We emphasize that our results were obtained from the Schrödinger equation describing the dynamical evolution of the entire system, without explicit use of the projection postulate. This is in contrast with other works, as for instance [6], where the reduction was repeatedly involved during the continuous measurement process. Although our final result does not display any slowing down of the decay rate, it should not be considered as a contradiction with the projection postulate. Indeed, the hindering of the decay rate, generated by the projection postulate, relies on the assumption that the probability of transitions between different quantum states is $Q(\Delta t)=a(\Delta t)^{2}$. It is definitely correct for transitions between isolated states, where the transition probability has an oscillatory behavior. However, in the case of transitions from isolated to very dense states, $Q(t)$ represents a sum of many oscillations with close frequencies. When averaged over the time-interval $\delta t=1 / \overline{\mathcal{E}}$, where $\overline{\mathcal{E}}$ is the width of the function $|\Omega(E)|^{2} \rho(E)$ (in our case $\overline{\mathcal{E}} \rightarrow \infty$ ), the resulting $Q(t)$ would then represent a pure exponential decay. In this case $Q(\Delta t) \propto \Delta t$, so that repeated applications of the projection postulate would not change the life-time of the decayed state. But, if $\delta t$ is finite that zero, due to the energy dependence of $\rho$ or $\Omega$, then $\sigma_{00}(t)$ exhibits deviations from the exponential behavior, which could result in quantum Zeno effect [6].

In conclusion, we have given a microscopic description of quantum Zeno effect in pure exponentially decaying quantum systems including the measurement devices. Our results show that while the measurements does not affect the decay rate, the energy distribution of the tunneling electron is broadened. This description applies to a wide range of physical processes as mentioned at the beginning of this letter. In particular, it can be verified in experiments with mesoscopic quantum dots, by using the point-contact detector [11], or an alternative set up.

We thank S. Levit and Y. Imry for most valuable discussions. One of us (S.G.) would like to acknowledge the hospitality of Oak Ridge National Laboratory and TRIUMF, while parts of this work were being performed. One of us (B.E.) gratefully acknowledge the support of GIF and the Israeli Ministry of Science and Technology and the French Ministry of Research and Technology

[1] B. Misra and E.C.G. Sudarshan, J. Math. Phys. 18 (1977).

[2] J. von Neumann, "Mathematische Grundlagen der Quantentheorie", Springer, Berlin, 1931.

[3] W.M. Itano, D.J. Heinzen, J.J. Bollinger, and D.j. Vineland, Phys. Rev. A41, 2295 (1990); C. Presilla, R. Onofrio and U. Tambini, Ann. of Phys. 248 (1996), 95, and references therein.

[4] R.A. Harris and L. Stodolsky, Phys. Lett. B116 (1982), 464; E. Block and P.R. Berman, Phys. Rev. A44, 1466 (1991). V. Frerichs and A. Schenzle, Phys. Rev. A44, 1962 (1991).

[5] S.A. Gurvitz, Phys. Rev. B56, 15215 (1997).

[6] L. Fonda, G. C. Ghirardi and A. Rimini, Rep. Prog. Phys. 41, 587, (1978).

[7] L.S. Schulman, Phys. Rev. A57, 1509 (1998).

[8] A.G. Kofman and G. Kurizki, Phys. Rev. A54, R3750 (1996).

[9] A. D. Panov, Phys. Lett. A260, 441 (1999), and references therein.

[10] R. Landauer, J. Phys. Condens. Matter 1, 8099 (1989).

[11] M. Field et al., Phys. Rev. Let. 70, 1311 (1993); E. Buks, R. Shuster, M. Heiblum, D. Mahalu and V. Umansky, Nature 391, 871 (1998).

[12] S.A. Gurvitz and Ya.S. Prager, Phys. Rev. B53 (1996), 15932; S.A. Gurvitz, Phys. Rev. B57 (1998) 6602.

[13] G. Hackenbroich, B. Rosenow, and H.A. Weidenmüller, Phys. Rev. Lett. 81, 5896 (1998).

[14] B. Elattari and S.A. Gurvitz (unpublished) 